\title{
Low permanent pacemaker rates following Lotus device implantation for transcatheter aortic valve replacement due to modified implantation protocol
}

\author{
Florian Krackhardt ${ }^{1}$, Behrouz Kherad ${ }^{1}$, Maximilian Krisper ${ }^{1}$, \\ Burkert Pieske ${ }^{1,2,3}$, Michael Laule ${ }^{4}$ Carsten Tschöpe ${ }^{1,3,5}$ \\ ${ }^{1}$ Department of Cardiology, Charité - Universitätsmedizin Berlin, \\ Campus Virchow Klinikum, Berlin, Germany \\ ${ }^{2}$ The German Center for Cardiovascular Disease (DZHK), Berlin, Germany \\ ${ }^{3}$ Department of Cardiology, Deutsches Herzzentrum Berlin (DHZB), Berlin, Germany \\ ${ }^{4}$ Department of Cardiology, Charité - Universitätsmedizin Berlin, Campus Mitte, Berlin, Germany \\ ${ }^{5}$ Berlin Center for Regenerative Therapies (BCRT), Berlin, Germany
}

\begin{abstract}
Background: Conduction disturbances requiring permanent pacemaker implantation following transcatheter aortic valve replacement (TAVR) are a common problem. Pacemaker implantation rates after TAVR appear to be higher compared to conventional aortic valve replacement. The aim of this study was to analyze whether a high annulus implantation conveys the benefit of a decreased rate of permanent pacemaker implantation while being safe and successful according to Valve Academic Research Consortium 2 (VARC2)-criteria.
\end{abstract}

Methods: A total of 23 patients with symptomatic severe aortic valve stenosis, an aortic annulus of 19-27 $\mathrm{mm}$ and at high risk for surgery were treated with the Lotus valve. In all patients the valve was implanted in a high annulus position via femoral access. The primary device performance endpoint was VARC2-defined device success after 30 days and the primary safety endpoint was the need for permanent pacemaker implantation.

Results: The mean age was $73.23 \pm 7.65$ years, $46 \%$ were female, $38 \%$ were New York Heart Association class III/IV at baseline. Thirty-day follow-up data were available for all patients. The VARC2-defined device success rate after 30 days was 22/23 (96\%). 2/21 (10\%) patients required a newly implanted pacemaker due to $3^{\text {rd }}$ degree atrioventricular block. $25 \%$ of the patients developed a new left bundle branch block after valvuloplasty or device implantation. 21 of the 23 patients (96\%) had no other signs of conduction disturbances after 30 days.

Conclusions: The approach of the modified implantation technique of Lotus TAVR device was safe and effective. The incidence of need for a permanent pacemaker following TAVR could be significantly reduced due to adopted implantation protocol. (Cardiol J 2017; 24, 3: 250-258)

Key words: aortic stenosis, transcatheter aortic valve replacement, permanent pacemaker, conduction disturbances, atrioventricular block

Address for correspondence: Florian Krackhardt, MD, Department of Cardiology, Charité — Universitätsmedizin Berlin, Campus Virchow Klinikum, Augustenburger Platz 1, 13353 Berlin, Germany, tel: 030/450-565407, e-mail: florian.krackhardt@charite.de

Received: 13.07.2016 Accepted: 03.01.2017 


\section{Introduction}

Transcatheter aortic valve replacement (TAVR) has proved to be a safe and effective treatment for severe aortic stenosis in appropriately selected high and extremely high surgical risk patients $[1,2]$. Since its introduction in 2002 [3], TAVR has gained wide acceptance and implant rates have grown immensely [4].

In recent years an increasing number of prosthetic valves have entered clinical trials and routine practice [5-7]. Most of these devices incorporate novel features designed to reduce the pitfalls identified with current-generation devices. The Lotus prosthetic valve (Boston Scientific, USA) is a second-generation TAVR device that employs a novel expansion mechanism. The outer surface of the lower half of the frame is covered with an adaptive seal, which seals the frame against the native aortoventricular interface $[7,8]$. The device is fully repositionable and resheathable, even in the completely expanded position, allowing for fine control and potential for removal.

The REPRISE I Trial (Repositionable Percutaneous Replacement of Stenotic Aortic valve Through Implantation of Lotus-Valve System) was a proof-of-concept study with the Lotus valve for TAVR with a procedural success rate of $100 \%$ $(n=11)$ [9]. The REPRISE II study showed that the valve was successfully implanted in all patients, with no cases of valve embolization, ectopic valve deployment, or additional valve implantation. All repositioning $(\mathrm{n}=26)$ and retrieval $(\mathrm{n}=6)$ attempts were successful. At 30 days, the mortality rate was $4.2 \%$, and the rate of disabling stroke was $1.7 \% ; 1(1.0 \%)$ patient had moderate paravalvular regurgitation, whereas none had severe paravalvular regurgitation thereby demonstrating safety and efficacy of this new device [10].

The occurrence of conduction abnormalities leading to the need for permanent pacemaker implantation (PPI) remains one of the most concerning complications associated with TAVR, with an incidence ranging from $9.3 \%$ to $42 \%$ among patients receiving a self-expandable valve (CoreValve, Medtronic, USA) [11, 12] and from 2.5\% to $11.5 \%$ after TAVR with a balloon-expandable valve (Edwards valve, Edwards Lifesciences, USA) $[13,14]$. Conduction disturbances also pose a well-known problem after surgical aortic valve replacement, with an incidence ranging from $3.2 \%$ to $8.5 \%[15,16]$.

For the Lotus valve, the REPRISE I study showed that $36 \%$ of patients (4/11) required a permanent pacemaker post-procedure [9], whereas $28.6 \%$ of patients $(34 / 120)$ received a permanent pacemaker in the REPRISE II study [10]. A number of factors have been attributed as predictors of PPI, including depth of implantation.

The aim of this study was to analyze if a high annulus implantation conveys the benefit of a decreased rate of PPI while being safe and successful according to the Valve Academic Research Consortium (VARC2)-criteria.

\section{Methods}

A total of 23 patients with symptomatic severe aortic stenosis were included in this study. All patients were treated at a single German center. Patients were eligible for inclusion if they had severe aortic stenosis based on echocardiographic criteria and reported symptoms attributable to severe aortic stenosis. Decision on interventional strategy, access route and selection of the device was done in a Heart Team session on Charité, Campus Virchow Klinikum.

The study was approved by the local ethics committee and all patients gave written informed consent.

\section{Pre-procedural echocardiographic assessment}

Echocardiography was performed using a Vivid-7 machine (GE Healthcare, USA). All scans were assessed by an experienced echocardiologist with severity of aortic stenosis graded based on European Association of Echocardiography and American Society of Echocardiography joint guidelines [17].

\section{Valve implantation}

All TAVR procedures were performed in a hybrid operation room with patients under general anesthesia. Two cardiologists (F.K. and M.L.) performed all procedures with both operators present at each procedure.

The femoral artery was used for device access in all cases with an 18-F Lotus Introducer (Boston Scientific) used for 23-mm Lotus cases and 20-F Lotus Introducer for those receiving a $27-\mathrm{mm}$ Lotus valve. The femoral access was routinely "pre-closed" with 2 Proglide devices (Abbott Vascular, Abbott Park, Illinois), and final closure was performed using a crossover balloon occlusion technique [18].

Balloon valvuloplasty was performed in 12 out of 23 patients (52\%) under rapid ventricular pacing to enable maximal balloon stability. Deployment of 


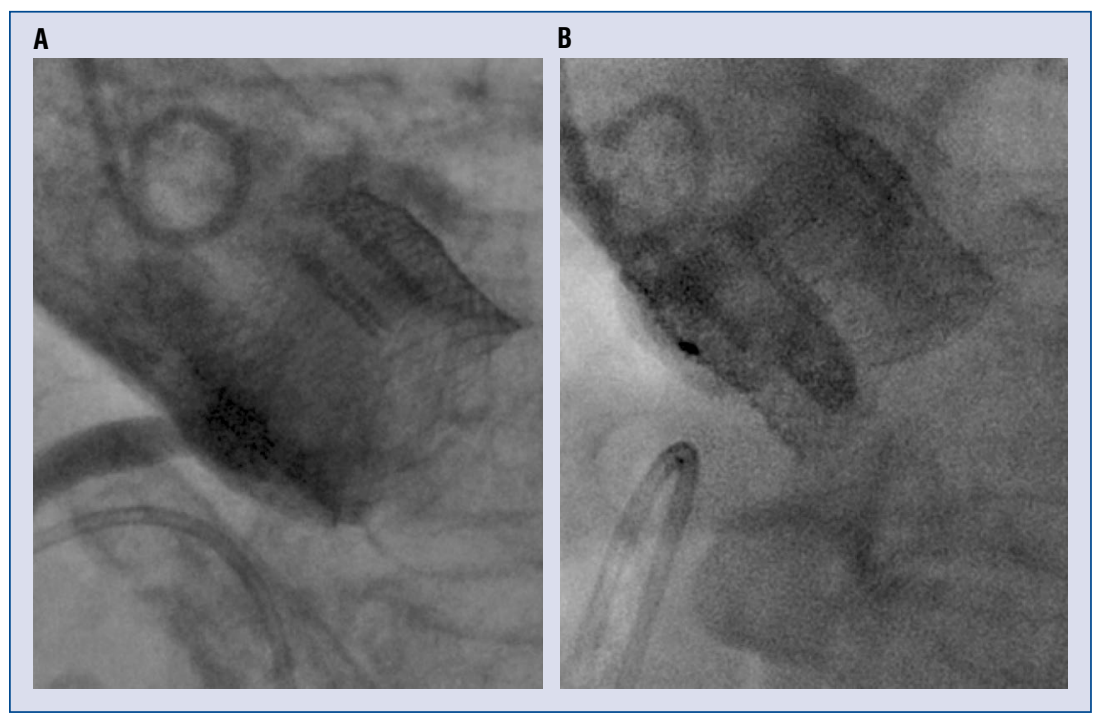

Figure 1. Modified implantation technique of the Lotus aortic bioprosthesis, showing higher seating (A) versus conventional implantation (B).

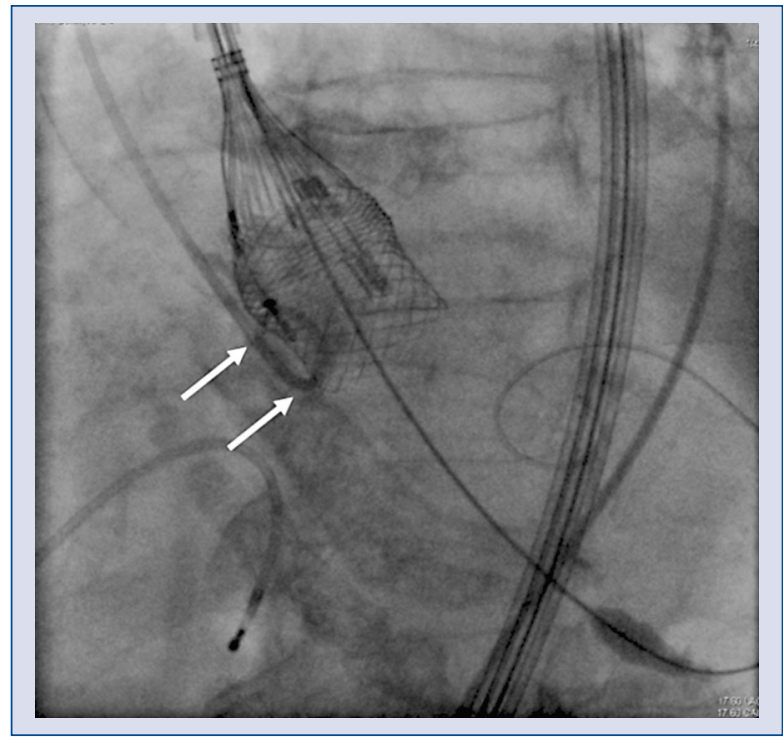

Figure 2. High positioning of the Lotus device. The radiopaque marker of the superior portion of the Lotus device is aligned with the upper curve of the pigtail catheter, which is seated in the aortic cusp (arrows).

the respective devices was performed in accordance with manufacturer's guidelines and best practices $[7,8,19]$. Regarding the implantation marker of the device, Lotus valves were implanted in this trial as high as possible with the same pull and push technique described previously [7, 8]. The Lotus aortic bioprosthesis has a radiopaque marker, which should be used as a reference point to facilitate orientation. For regular implantation regime the marker should be in close proximity to the pigtail catheter in the aortic root. In this modified implantation protocol, the radiopaque marker was positioned above the pigtail catheter curve at the beginning of valve deployment. This ensures a higher positioning of approximately $4 \mathrm{~mm}$ or less (Figs. 1, 2). Aortic regurgitation was assessed by aortography after final deployment. Moderate or greater aortic regurgitation, identified at the time of deployment was treated by repositioning.

\section{Post-procedural echocardiographic assessment}

All patients had a transthoracic echocardiography study performed post-procedural and at 30 day follow-up. The post-procedural assessment included prosthesis function, degree and location of aortic regurgitation and left ventricular function. Prosthetic regurgitation was assessed in accordance with VARC2 [20] recommendations.

\section{Clinical review}

A study investigator reviewed patients at the time of each echocardiogram, and continuous recording of atrioventricular (AV) conduction for $72 \mathrm{~h}$ post procedure were performed and a detailed history was taken and an examination performed. New York Heart Association functional class was determined on the basis of the patient's selfreporting of symptoms. Rhythm monitoring was done continuously via monitor in an intensive care unit or telemetry on the cardiology ward. 
Table 1. Demographic data and clinical characteristics of the patient population.

\begin{tabular}{lc}
\hline Parameter & Mean \pm SD or $\mathbf{n ~ ( \% ) ~}$ \\
\hline Age [years] & $76.13 \pm 8.18$ \\
Male & $9(39 \%)$ \\
STS Risk Score & $19.99 \pm 7.69$ \\
log EUROscore [\%] & $15.01 \pm 11.52$ \\
NYHA functional class & \\
II & $8 \pm 34.78$ \\
III & $12 \pm 52.17$ \\
IV & $3 \pm 13.04$ \\
Hypertension & $22(95.65 \%)$ \\
Diabetes & $7(30.43 \%)$ \\
Coronary artery disease & $16(69.57 \%)$ \\
History of PCI & $7 \pm 30.43$ \\
History of CABG & $4 \pm 17.39$ \\
History of atrial fibrillation & $5 \pm 21.74$ \\
\hline
\end{tabular}

STS - the Society of Thoracic Surgeons risk of morbidity or mortality score; NYHA - New York Heart Association; $\mathrm{PCl}$ - percutaneous coronary intervention; CABG - coronary artery bypass grafting

Table 2. Key parameters evaluated at initial echocardiographic assessment.

\begin{tabular}{lc}
\hline Parameter & Mean \pm SD \\
\hline Transaortic gradient $[\mathrm{mm} \mathrm{Hg}]$ & $42.95 \pm 18.23$ \\
Aortic velocity $[\mathrm{m} / \mathrm{s}]$ & $4.07 \pm 0.88$ \\
Aortic valve area $\left[\mathrm{cm}^{2}\right]$ & $0.73 \pm 0.13$ \\
Aortic root $[\mathrm{mm}]$ & $24.87 \pm 1.71$ \\
Aortic regurgitation & $1.06 \pm 0.73$ \\
LV-function - LVEF [\%] & $50.00 \pm 13.84$ \\
\hline
\end{tabular}

LV — left ventricular; LVEF — left ventricular ejection fraction; $\mathrm{SD}$ - standard deviation

\section{Clinical endpoints}

The primary clinical endpoint of the trial was VARC2-defined device success [20]. Prosthetic function was determined by core laboratory assessment of the discharge echocardiogram. Secondary endpoints were all-cause and cardiovascular mortality at 30 days, minor and major bleeding, minor and major vascular injury and due to modified implantation technique secondary endpoints which included conduction disturbances and pacemaker implantation rates post TAVR implantation.

\section{Results}

Within 6 months 23 patients were treated with a Lotus device using a modified implantation technique at this center. Table 1 shows baseline characteristics. The mean Society of Thoracic Surgeons risk of morbidity or mortality score was $19.99 \pm 7.69$. All patients were discussed by the Heart Team and were considered to be high risk for surgery. Table 2 shows echocardiographic assessment before the index procedure.

\section{Procedural success and clinical outcome}

Lotus valve implantation was successful in all patients. Ten $23 \mathrm{~mm}$, nine $25 \mathrm{~mm}$ and four $27 \mathrm{~mm}$ Lotus valves were implanted. Partial recapture was necessary in $52 \%$ of the cases (12 patients).

\section{Clinical outcomes}

According to REPRISE I and II data clinical procedural success rate and device success rate were $100 \%$, all devices were implanted by transfemoral access. Delivery, deployment, valve positioning, delivery system retrieval were successful in $100 \%$ too. In all cases intended valve performance was $100 \%$, no major adverse cardiac and cerebrovascular events (MACCE) occurred through discharge. One patient died at day 28 due to a pneumonia and sepsis in an external rehabilitation hospital and there were two minor strokes during the 30 day-follow up. There were no major vascular complications. Three (13\%) patients experienced minor bleeding at the closure site, no patient required surgery or blood transfusions (Table 3 ).

\section{Echocardiography outcome at discharge and at follow-up}

Echocardiographic assessment postprocedural showed a significant decrease in mean aortic gradient (before $42.09 \pm 18.23 \mathrm{~mm} \mathrm{Hg}$ vs. postprocedural $13.96 \pm 4.12 \mathrm{~mm} \mathrm{Hg}, \mathrm{p}<0.001$ ) and a significant increase in effective orifice area (before $0.73 \pm 0,13 \mathrm{~cm}^{2}$ vs. postprocedural $1.52 \pm 0.25$ $\mathrm{cm}^{2}, \mathrm{p}<0.001$ ) (Tables 2, 4).

\section{VARC2 criteria}

Severe paravalvular aortic regurgitation was not detected in any of the patients, neither after the procedure nor at 30 day follow-up. No patient had paravalvular aortic regurgitation which was more than trivial (Fig. 3).

The average mean aortic valve gradient was $13.96 \pm 4.12 \mathrm{~mm} \mathrm{Hg}$ and a mean aortic valve gradient of more than $20 \mathrm{~mm} \mathrm{Hg}$ was only observed in $1 / 23$ patients after the procedure and during 30 day follow-up (Table 4).

The mean effective orifice area after the procedure was $1.57 \pm 0.25 \mathrm{~cm}^{2}$ and no patient/valve mismatch as defined by the VARC2 criteria was observed (Table 4). 
Table 3. Outcomes and clinical endpoint at discharge and at 30 day follow up.

\begin{tabular}{lcc}
\hline & $\begin{array}{c}\text { At discharge } \\
(\%)\end{array}$ & $\begin{array}{c}\text { At follow-up } \\
(30 \text { days })(\%)\end{array}$ \\
\hline Major adverse cardiac and cerebrovascular events & $0(0 \%)$ & $1(4.35 \%)$ \\
Death & $0(0 \%)$ & $1(4.35 \%)$ \\
Myocardial infarction $<72 \mathrm{~h}$ & $0(0 \%)$ & $0(0 \%)$ \\
Major stroke & $0(0 \%)$ & $0(0 \%)$ \\
Urgent conversion to surgery or repeat procedure & $0(0 \%)$ & $0(0 \%)$ \\
for valve-related dysfunction & $2(8.6 \%)$ & $0(0 \%)$ \\
Vascular complications & $2(8.6 \%)$ & $0(0 \%)$ \\
Minor bleeding & $0(0 \%)$ & $0(0 \%)$ \\
Acute kidney injury & $2(8.7 \%)$ & \\
Myocardial infarction $>72 \mathrm{~h}$ & & \\
Minor stroke or transient ischemic attack & & \\
\hline
\end{tabular}

Table 4. Key echocardiographic parameters evaluated at discharge and at 30 day follow-up.

\begin{tabular}{lccc}
\hline Parameter & $\begin{array}{c}\text { At discharge } \\
\pm \text { standard deviation }\end{array}$ & $\begin{array}{c}\text { At follow-up (30 days) } \\
\pm \text { standard deviation }\end{array}$ & P \\
\hline Peak aortic gradient $[\mathrm{mm} \mathrm{Hg}]$ & $29.54 \pm 11.66$ & $28.34 \pm 10.55$ & $>0.05$ \\
Mean aortic gradient $[\mathrm{mm} \mathrm{Hg}]$ & $13.96 \pm 4.12$ & $12.64 \pm 3.75$ & $>0.05$ \\
Effective orifice area $\left[\mathrm{cm}^{2}\right]$ & $1.57 \pm 0.25$ & $1.62 \pm 0.23$ & $>0.05$ \\
Left ventricular ejection fraction $[\%]$ & $53.37 \pm 7.79$ & $51.91 \pm 10.21$ & $>0.05$ \\
Aortic regurgitation & $0.33 \pm 0.49$ & $0.0625 \pm 0.25$ & $>0.05$ \\
\hline
\end{tabular}

Intra- and interventricular conduction disturbances

In the initial electrocardiogram (ECG) 19 out of the 22 patients $(78.3 \%)$ showed sinus rhythm, whereas 5 of the patients $(21.7 \%)$ displayed atrial fibrillation. The mean $\mathrm{QRS}$ duration before the TAVR was $110.8 \pm 25.83 \mathrm{~ms}$ and $126.9 \pm 19.05 \mathrm{~ms}(\mathrm{p}>0.05)$ at discharge. It did not change significantly at the 30 day follow-up (QRS $125.9 \pm 13.03 \mathrm{~ms}, \mathrm{p}>0.05)$. The mean PR duration before the TAVR was $177.4 \pm 33.84 \mathrm{~ms}$ and at discharge to $169.8 \pm 47 \mathrm{~ms}$ $(\mathrm{p}>0.05)$ and did not change significantly at the 30 day follow-up (QRS $166.9 \pm 32.05 \mathrm{~ms}, \mathrm{p}>0.05$ ). $17.4 \%$ of the patients showed a left bundle branch block (LBBB) before the procedure and $25 \%$ of the patients displayed LBBB after the TAVR $(\mathrm{p}<0.05)$. One patient (13\%) showed a right bundle branch block (RBBB) in the initial ECG and that patient underwent elective pacemaker implantation before the TAVR. Post-TAVR there were no new RBBB observed. $17.4 \%$ of the patients displayed a first degree AV block at the initial presentation, whereas after TAVR there were only $15 \%$ first degree AV blocks ( $p>0.05$ ). One patient developed a third degree AV block after the
TAVR and required the implantation of a permanent pacemaker (Fig. 4).

\section{Statistical analysis}

Continuous data were presented as mean \pm standard deviation (SD) and dichotomous data in percentage. Differences in continuous variables between two groups were analyzed using Student's t-test. All statistical analyses were performed with Statview 5.0 (SAS Institute), SPSS 22.0 (IBM) and PRISM 5.0 (GraphPad). Differences were considered statistically significant when $\mathrm{p}<0.05$.

\section{Discussion}

There is growing evidence supporting the efficacy and safety of TAVR as an alternative treatment strategy to surgical valve replacement in patients at higher-risk $[1,10]$ and its superiority to medical therapy in patients denying surgery [2].

Despite improvements in deployment techniques, a number of limitations remained, including vascular access complications [21, 22], the need for permanent pacemaker after implantation 


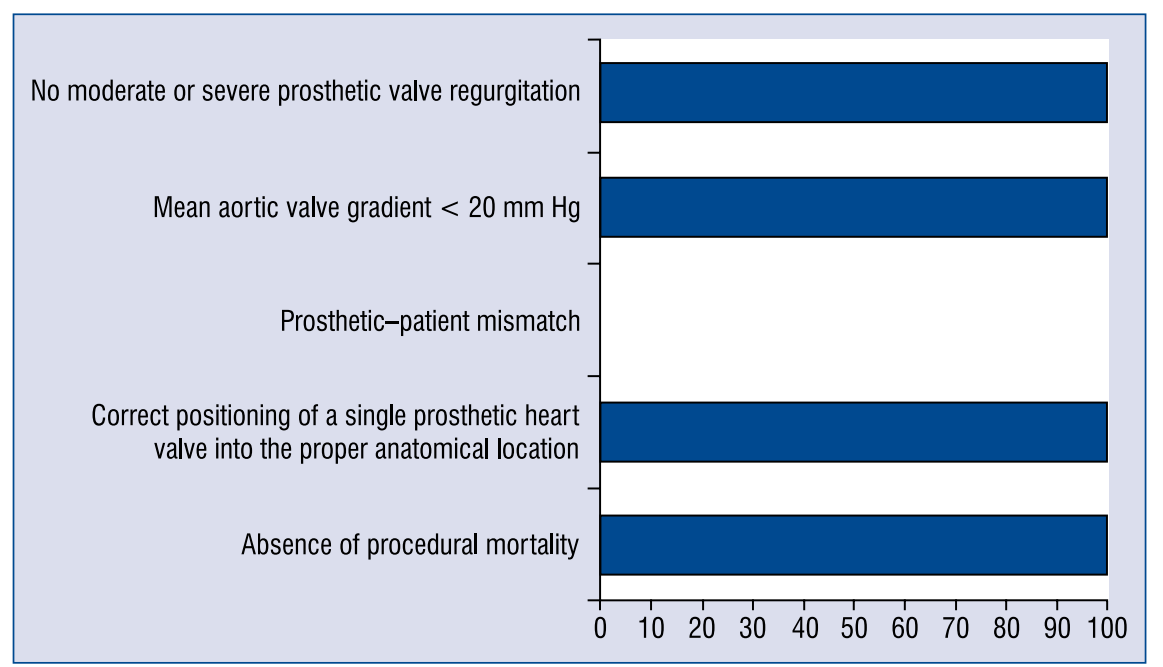

Figure 3. Procedural success as determined by VARC2 criteria.

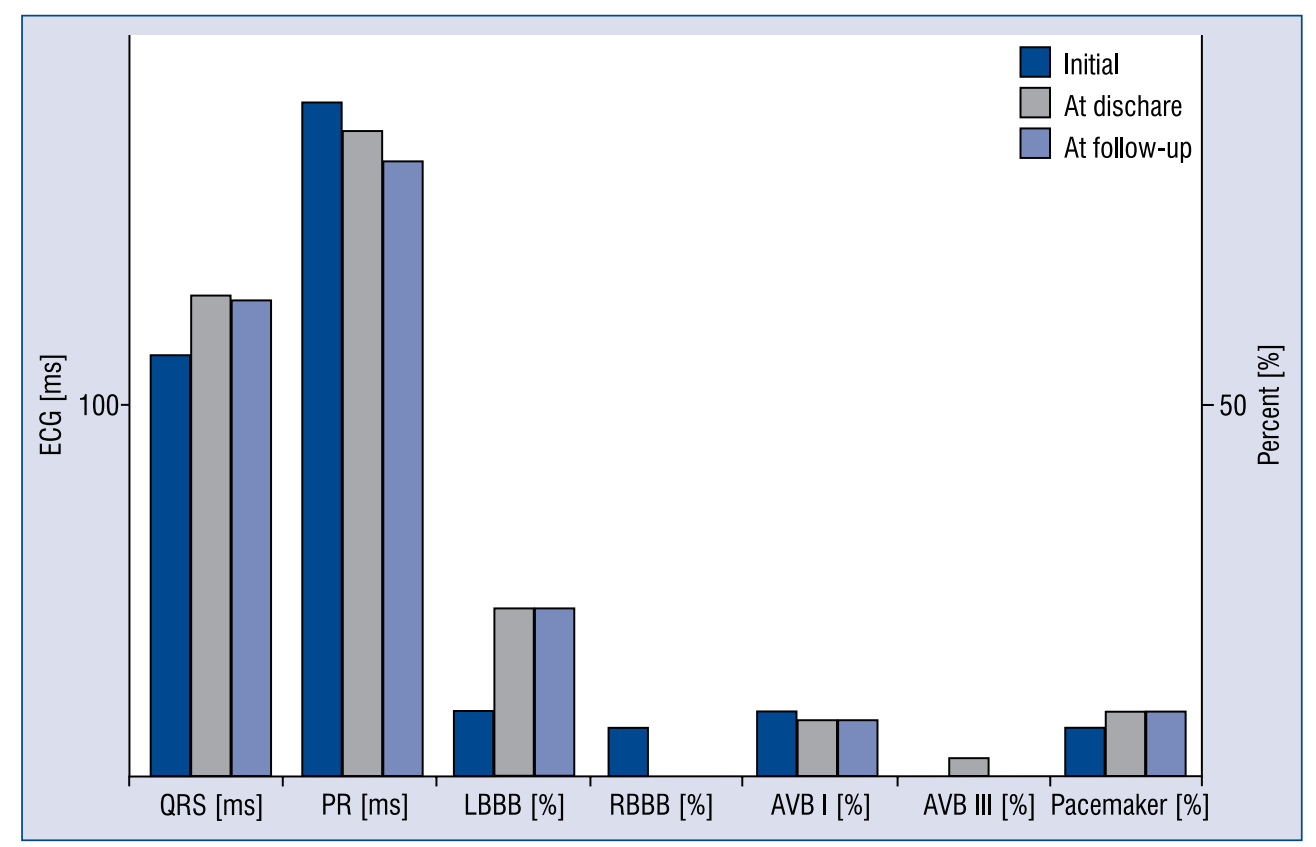

Figure 4. QRS duration, new onset of bundle branch block and rate of high grade atrioventricular block (AVB) before and after procedure; LBBB — left bundle branch block; RBBB — right bundle branch block.

$[11,23]$, periprosthetic aortic regurgitation [24] and stroke [25, 26].

The Lotus valve has been shown to be highly efficient with high rates of procedural success, driven by high rates of correct positioning of a single device and lower rates of moderate paraprosthetic aortic regurgitation (PAR) [7]. The Lotus valve has also shown to be safe with low rates of procedural mortality and low rates of transprosthetic gradients greater than $20 \mathrm{~mm} \mathrm{Hg}$ [7].
However, published data suggests that the Lotus valve has a higher rate of permanent pacemaker insertion after TAVR implantation [9-11]. Higher rates of pacemaker implantation have been shown to be associated with depth of implantation and the size of the prosthetic device [27]. It has been shown that a higher seating in regard to the aortic annulus reduces the need for PPI [28, 29].

In this study, analysis of whether a higher implantation technique of the Lotus valve is safe 
and successful but also, whether it decreases the requirement of pacemaker implantation.

\section{Higher implantation of the Lotus valve} shows similar efficacy and safety

In this single-center non-randomized trial the modified Lotus device implantation was associated with high rates of procedural success $(100 \%)$. This result is identical to the procedural success rate of $100 \%$ reported by Gooley et al. [30] and the procedural success rate of $100 \%$ reported in both the REPRISE I and REPRISE II trials [9, 10].

The modified implantation technique also showed high VARC2-defined primary composite outcome of device success of $79 \%$. These results are similar to the VARC2-as defined by a success rate of $84 \%$ reported by Gooley et al. [30] and even higher compared to VARC2-defined success rate of $60.7 \%$ reported in the REPRISE II trial [10].

The rate of moderate or severe PAR in this study was $0 \%$. Again this result is similar to the percent of moderate to severe PAR of $0 \%$ reported in both the REPRISE I and REPRISE II trials [9, 10].

The observed MACCE rate in this study of $4.35 \%$ is low and similar to the MACE rates of $9.1 \%$ and $4.2 \%$ reported in the REPRISE I and REPRISE II trials, respectively $[9,10]$.

Two out of the 23 patients $(8.7 \%)$ had a minor stroke following TAVI in this study. This is slightly higher than the stroke rates of $1.7 \%$ reported by Gooley et al. [30] and the stroke rates of $0 \%$ and $4 \%$ reported in the REPRISE I and REPRISE II trials, respectively $[9,10]$.

\section{Intraventricular conduction impairment}

Houthuizen et al. [31] reported that approximately $40 \%$ of patients developed a new LBBB after TAVR of which most persisted at follow-up, however without the need for pacemaker implantation. In another analysis, a proportion of $\mathrm{AV}$ conduction disturbances after the intervention has been shown to recover at 3 months of follow-up, and only $40 \%$ of the permanent pacemaker patients for highdegree AV block still had an AV block underlying their paced rhythm [32]. Without any clinical signs for pacemaker requirements our approach to wait and observe has been safe in our patient population. Due to the relatively low sample size of these studies, this issue needs further investigation.

\section{Reduced rate of AV-conduction impairment} and therefore decreased pacemaker rates

The observed rate of AV-conduction impairment of second or third degree post TAVR was
$9.5 \%(2 / 21)$ in this study. Subsequently, pacemaker implantation rate was also $9.5 \%(2 / 21)$ in this study, which is markedly lower than the previous published rates of $28 \%$ [30] and the rates reported of $36 \%$ and $28.6 \%$ in the REPRISE I and REPRISE II studies, respectively [9, 10].

Pre-existing RBBBs have been shown to be a strong predictor for higher AV-conduction impairment and requirement of PPI after TAVR and it is usually recommended to implant pacemakers in those patients before the procedure [33].

Both patients requiring PPI post TAVR due to third degree AV conduction impairment in this study displayed a RBBB in the initial ECG. In both patients the clinical situation was critical so it was decided to proceed with the TAVR knowing that there was a high risk of postprocedural requirement of a PPI.

In this study $43.48 \%$ of the patients underwent TAVR using a $23 \mathrm{~mm}$ prosthetic valve, $39.13 \%$ using a $25 \mathrm{~mm}$ prosthetic valve and $17.39 \%$ using a $27 \mathrm{~mm}$ valve. For the REPRISE I trial there was only one valve size $(23 \mathrm{~mm})$ available and for the REPRISE II study only the 23 and $27 \mathrm{~mm}$ Lotus valve were available $[9,10]$. In the study by Gooley et al. [30] $52 \%$ of the patient had a $23 \mathrm{~mm}$ Lotus prosthetic valve and $48 \%$ had a $27 \mathrm{~mm}$ prosthetic valve.

The risk of postoperative AV block has been shown to increase by 2 -fold following a large valve implantation in a small annulus, 4 -fold using CoreValve versus the Sapien valve [34]. Moreover, large meta-analyses have shown that oversizing $>4 \mathrm{~mm}$ is a major risk factor for PPI following TAVI [35]. The smaller bioprosthetic valves used in our study might therefore have contributed to the lower rate of PPI required in our patient population.

The implantation technique used during the REPRISE trials was markedly deeper within the left ventricular outflow tract comparable to the first techniques used for the CoreValve. Piazza et al were able to show that implantation depth was $10.3 \mathrm{~mm}$ in those patients who required a PPI, versus $5.5 \mathrm{~mm}$ in those without PPI requirement [36]. Similar findings were shown for implantation of balloon-expandable TAVR [28].

The potential pitfalls of higher implantation technique are obstruction of the coronary arteries and a higher risk of valve dislocation thereby making a second prosthetic valve implantation necessary.

A low position of the coronary ostia with respect to the aortic annulus has been highlighted as one of the most important factors contributing to 
obstruction of the coronary ostia following TAVR, and it has been suggested that a coronary ostia height of $\leq 10 \mathrm{~mm}$ increases the risk of coronary obstruction during TAVR [37-39].

The principal findings of the present study are:

- higher implantation of the Lotus valve shows similar efficacy and safety characteristics compared to the lower implantation technique described in the REPRISE trials;

- TAVI using the Lotus bioprothetic valve impairs intraventricular conduction resulting in a new left bundle block in $25 \%$ of the patients;

- higher graded AV-conduction abnormalities following TAVR implantation can be markedly reduced by exact valve sizing and a high implantation technique relative to the aortic annulus thereby decreasing the pacemaker rates to a level comparable to TAVR with a balloon expanding valve or surgical aortic valve replacement.

\section{Conclusions}

The approach of a high implantation technique of Lotus TAVR devices was safe and effective. The incidence of the need for a permanent pacemaker following TAVR could be significantly reduced due to the modified implantation protocol.

\section{Conflict of interest: None declared}

\section{References}

1. Smith CR, Leon MB, Mack MJ, et al. PARTNER Trial Investigators. Transcatheter versus surgical aortic-valve replacement in high-risk patients. N Engl J Med. 2011; 364(23): 2187-2198, doi: 10.1056/NEJMoa1103510, indexed in Pubmed: 21639811.

2. Makkar RR, Fontana GP, Jilaihawi H, et al. PARTNER Trial Investigators. Transcatheter aortic-valve replacement for inoperable severe aortic stenosis. N Engl J Med. 2012; 366(18): 1696-1704, doi: 10.1056/NEJMoa1202277, indexed in Pubmed: 22443478.

3. Cribier A, Eltchaninoff H, Bash A, et al. Percutaneous transcatheter implantation of an aortic valve prosthesis for calcific aortic stenosis: first human case description. Circulation. 2002; 106(24): 3006-3008, indexed in Pubmed: 12473543.

4. Osnabrugge RLJ, Mylotte D, Head SJ, et al. Aortic stenosis in the elderly: disease prevalence and number of candidates for transcatheter aortic valve replacement: a meta-analysis and modeling study. J Am Coll Cardiol. 2013; 62(11): 1002-1012, doi: 10.1016/j.jacc.2013.05.015, indexed in Pubmed: 23727214.

5. Binder RK, Schäfer U, Kuck KH, et al. Transcatheter aortic valve replacement with a new self-expanding transcatheter heart valve and motorized delivery system. JACC Cardiovasc Interv. 2013; 6(3): 301-307, doi: 10.1016/j.jcin.2013.01.129, indexed in Pubmed: 23517843.

6. Freeman M, Rodés-Cabau J, Urena M, et al. First-in-man transfemoral transcatheter aortic valve replacement with the 29 mm Edwards SAPIEN XT valve. Catheter Cardiovasc Interv. 2013; 82(4): 664-670, doi: 10.1002/ccd.24543, indexed in Pubmed: 22744829 .

7. Gooley R, Lockwood S, Antonis P, et al. The SADRA Lotus Valve System: a fully repositionable, retrievable prosthesis. Minerva Cardioangiol. 2013; 61(1): 45-52, indexed in Pubmed: 23381379.

8. Meredith IT, Hood KL, Haratani N et al. Boston Scientific Lotus valve. EuroIntervention. 2012 Sep;8 Suppl Q:Q70-4. doi: 10.4244/EIJV8SQA12.

9. Meredith IT, Worthley SG, Whitbourn RJ, et al. Transfemoral aortic valve replacement with the repositionable Lotus Valve System in high surgical risk patients: the REPRISE I study. EuroIntervention. 2014; 9(11): 1264-1270, doi: 10.4244/EIJV9I11A216, indexed in Pubmed: 24169077.

10. Meredith IT, Worthley SG, Whitbourn RJ, et al. Transfemoral aortic valve replacement with the repositionable Lotus Valve System in high surgical risk patients: the REPRISE I study. EuroIntervention. 2014; 9(11): 1264-1270, doi: 10.4244/EIJV9I11A216, indexed in Pubmed: 24169077.

11. Fraccaro C, Buja G, Tarantini G, et al. Incidence, predictors, and outcome of conduction disorders after transcatheter selfexpandable aortic valve implantation. Am J Cardiol. 2011; 107(5): 747-754, doi: 10.1016/j.amjcard.2010.10.054, indexed in Pubmed: 21247519.

12. Zahn R, Gerckens U, Grube E, et al. German Transcatheter Aortic Valve Interventions-Registry Investigators. Transcatheter aortic valve implantation: first results from a multi-centre realworld registry. Eur Heart J. 2011; 32(2): 198-204, doi: 10.1093/ eurheartj/ehq339, indexed in Pubmed: 20864486.

13. Lefèvre T, Kappetein AP, Wolner E, et al. PARTNER EU Investigator Group. One year follow-up of the multi-centre European PARTNER transcatheter heart valve study. Eur Heart J. 2011; 32(2): 148-157, doi: 10.1093/eurheartj/ehq427, indexed in Pubmed: 21075775 .

14. Godin M, Eltchaninoff H, Furuta A, et al. Frequency of conduction disturbances after transcatheter implantation of an Edwards Sapien aortic valve prosthesis. Am J Cardiol. 2010; 106(5): 707-712, doi: 10.1016/j.amjcard.2010.04.029, indexed in Pubmed: 20723650.

15. Boughaleb D, Mansourati J, Genet L, et al. [Permanent cardiac stimulation after aortic valve replacement: incidence, predictive factors and long-term prognosis]. Arch Mal Coeur Vaiss. 1994; 87(7): 925-930, indexed in Pubmed: 7702437.

16. Bagur R, Manazzoni JM, Dumont É, et al. Permanent pacemaker implantation following isolated aortic valve replacement in a large cohort of elderly patients with severe aortic stenosis. Heart. 2011; 97(20): 1687-1694, doi: 10.1136/heartjnl-2011-300308, indexed in Pubmed: 21828221.

17. Baumgartner H, Hung J, Bermejo J, et al. EAE/ASE. Echocardiographic assessment of valve stenosis: EAE/ASE recommendations for clinical practice. Eur J Echocardiogr. 2009; 10(1): 1-25, doi: 10.1093/ejechocard/jen303, indexed in Pubmed: 19065003.

18. Genereux P, Kodali S, Leon MB, et al. Clinical outcomes using a new crossover balloon occlusion technique for percutaneous closure after transfemoral aortic valve implantation. JACC Cardiovasc Interv. 2011; 4(8): 861-867, doi: 10.1016/j.jcin.2011.05.019, indexed in Pubmed: 21851899.

19. Linke A, Wenaweser P, Gerckens U, et al. ADVANCE study Investigators. Treatment of aortic stenosis with a self-expanding transcatheter valve: the International Multi-centre ADVANCE Study. Eur Heart J. 2014; 35(38): 2672-2684, doi: 10.1093/eurheartj/ehu162, indexed in Pubmed: 24682842. 
20. Kappetein AP, Head SJ, Généreux P, et al. Valve Academic Research Consortium-2. Updated standardized endpoint definitions for transcatheter aortic valve implantation: the Valve Academic Research Consortium-2 consensus document. J Thorac Cardiovasc Surg. 2013; 145(1): 6-23, doi: 10.1016/j.jtcvs.2012.09.002, indexed in Pubmed: 23084102.

21. Tchetche D, Dumonteil N, Sauguet A, et al. Thirty-day outcome and vascular complications after transarterial aortic valve implantation using both Edwards Sapien and Medtronic CoreValve bioprostheses in a mixed population. EuroIntervention. 2010; 5(6): 659-665, indexed in Pubmed: 20142215.

22. Van Mieghem NM, Nuis RJ, Piazza N, et al. Vascular complications with transcatheter aortic valve implantation using the 18 Fr Medtronic CoreValve System: the Rotterdam experience. EuroIntervention. 2010; 5(6): 673-679, indexed in Pubmed: 20142217.

23. van der Boon RM, Nuis RJ, Van Mieghem NM, et al. New conduction abnormalities after TAVI-frequency and causes. Nat Rev Cardiol. 2012; 9(8): 454-463, doi: 10.1038/nrcardio.2012.58, indexed in Pubmed: 22547171.

24. Sherif MA, Abdel-Wahab M, Stöcker B, et al. Anatomic and procedural predictors of paravalvular aortic regurgitation after implantation of the Medtronic CoreValve bioprosthesis. J Am Coll Cardiol. 2010; 56(20): 1623-1629, doi: 10.1016/j. jacc.2010.06.035, indexed in Pubmed: 21050971.

25. Nuis RJ, Van Mieghem NM, Schultz CJ, et al. Frequency and causes of stroke during or after transcatheter aortic valve implantation. Am J Cardiol. 2012; 109(11): 1637-1643, doi: 10.1016/j. amjcard.2012.01.389, indexed in Pubmed: 22424581.

26. Eggebrecht H, Schmermund A, Voigtländer T, et al. Risk of stroke after transcatheter aortic valve implantation (TAVI): a meta-analysis of 10,037 published patients. EuroIntervention. 2012; 8(1): 129-138, doi: 10.4244/EIJV8I1A20, indexed in Pubmed: 22391581.

27. Siontis GCM, Jüni P, Pilgrim T, et al. Predictors of permanent pacemaker implantation in patients with severe aortic stenosis undergoing TAVR: a meta-analysis. J Am Coll Cardiol. 2014; 64(2): 129-140, doi: 10.1016/j.jacc.2014.04.033, indexed in Pubmed: 25011716.

28. Binder RK, Webb JG, Toggweiler S, et al. Impact of post-implant SAPIEN XT geometry and position on conduction disturbances, hemodynamic performance, and paravalvular regurgitation. JACC Cardiovasc Interv. 2013; 6(5): 462-468, doi: 10.1016/j. jcin.2012.12.128, indexed in Pubmed: 23702010.

29. Ferreira ND, Caeiro D, Adão L, et al. Incidence and predictors of permanent pacemaker requirement after transcatheter aortic valve implantation with a self-expanding biopros- thesis. Pacing Clin Electrophysiol. 2010; 33(11): 1364-1372, doi: 10.1111/j.1540-8159.2010.02870.x, indexed in Pubmed: 20723083.

30. Gooley RP, Talman AH, Cameron JD, et al. Comparison of selfexpanding and mechanically expanded transcatheter aortic valve prostheses. JACC Cardiovasc Interv. 2015; 8(7): 962-971, doi: 10.1016/j.jcin.2015.03.014, indexed in Pubmed: 26088514.

31. Houthuizen P, van der Boon RMA, Urena M, et al. Occurrence, fate and consequences of ventricular conduction abnormalities after transcatheter aortic valve implantation. EuroIntervention. 2014; 9(10): 1142-1150, doi: 10.4244/EIJV9I10A194, indexed in Pubmed: 24273252.

32. Guetta V, Goldenberg G, Segev A, et al. Predictors and course of high-degree atrioventricular block after transcatheter aortic valve implantation using the CoreValve Revalving System. Am J Cardiol. 2011; 108(11): 1600-1605, doi: 10.1016/j.amjcard.2011.07.020, indexed in Pubmed: 21880290.

33. Roten L, Wenaweser P, Delacrétaz E, et al. Incidence and predictors of atrioventricular conduction impairment after transcatheter aortic valve implantation. Am J Cardiol. 2010; 106(10): 1473-1480, doi: 10.1016/j.amjcard.2010.07.012, indexed in Pubmed: 21059439.

34. Bleiziffer S, Ruge H, Hörer J, et al. Predictors for new-onset complete heart block after transcatheter aortic valve implantation. JACC Cardiovasc Interv. 2010; 3(5): 524-530, doi: 10.1016/j. jcin.2010.01.017, indexed in Pubmed: 20488409.

35. Schroeter T, Linke A, Haensig M, et al. Predictors of permanent pacemaker implantation after Medtronic CoreValve bioprosthesis implantation. Europace. 2012; 14(12): 1759-1763, doi: 10.1093/ europace/eus191, indexed in Pubmed: 22733983.

36. Piazza N, Onuma Y, Jesserun E, et al. Early and persistent intraventricular conduction abnormalities and requirements for pacemaking after percutaneous replacement of the aortic valve. JACC Cardiovasc Interv. 2008; 1(3): 310-316, doi: 10.1016/j. jcin.2008.04.007, indexed in Pubmed: 19463319.

37. Holmes D, Mack M, Kaul S, et al. 2012 ACCF/AATS/SCAI/STS expert consensus document on transcatheter aortic valve replacement. The Journal of Thoracic and Cardiovascular Surgery. 2012; 144(3): e29-e84, doi: 10.1016/j.jtcvs.2012.03.001.

38. Masson JB, Kovac J, Schuler G, et al. Transcatheter aortic valve implantation: review of the nature, management, and avoidance of procedural complications. JACC Cardiovasc Interv. 2009; 2(9): 811-820, doi: 10.1016/j.jcin.2009.07.005, indexed in Pubmed: 19778768.

39. Ribeiro HB, Nombela-Franco L, Urena M, et al. Coronary obstruction following transcatheter aortic valve implantation: a systematic review. JACC Cardiovasc Interv. 2013; 6(5): 452-461, doi: 10.1016/j.jcin.2012.11.014, indexed in Pubmed: 23602458. 